\title{
A note on the direct injection of turbulence by breaking waves
}

\author{
Nicolas Rascle ${ }^{a, b}$ *, Bertrand Chapron $^{a}$, Fabrice Ardhuin ${ }^{c}$, Alexander Soloviev ${ }^{d}$ \\ a Laboratoire d'Océanographie Spatiale, Institut Francais de Recherche pour l'Exploitation de la Mer, Plouzané, \\ France \\ ${ }^{\mathrm{b}}$ Nansen-Tutu Center, Department of Oceanography, University of Cape Town, Rondebosch, South Africa \\ ${ }^{c}$ Laboratoire Physique Hydrodynamique et Sédimentaire, Institut Francais de Recherche pour l'Exploitation de la \\ Mer, Plouzané, France \\ d Oceanographic Center, Nova Southeastern University, Dania Beach, FL, USA \\ *: Corresponding author : Nicolas Rascle, email address : tel.: +27 216505316 ; fax: +27 216503979 ; email \\ addresses : nrascle@ifremer.fr ; nicolas.rascle@ifremer.fr
}

\begin{abstract}
:
We investigate the turbulence induced by wave-breaking at the ocean surface. Two recent models use a mechanism of direct depth injection of turbulent kinetic energy (TKE) by breaking waves. Those models aim to reproduce the near-surface mean and turbulent properties, in particular the TKE dissipation rates. Of critical importance are the injection depth of each breaking wave and the size distribution of those breaking waves. The models by Sullivan et al. (2007) and by Kudryavtsev et al. (2008) have very different parameterizations, and those differences are reviewed here and compared to available observations. Using realistic parameterizations in these models leads to TKE injections too shallow to compare to observations, in particular for developed seas. The near-surface turbulence is thus still not well understood to the zeroth order. For instance, whether developed seas produce deeper or shallower mixing than young seas is neither well understood nor well modelled. Additional dedicated measurements as well as investigations of breaking non-breaking wave interactions are needed.
\end{abstract}

\section{Highlights}

Two models of the injection of turbulence by breaking waves are analyzed. They differ mainly in depth and size of their breaking waves. They are inconsistent with observed wave statistics and observed turbulence. Further developments are needed for a coherent description of the surface mixing.

Keywords: Surface waves ; Wave breaking ; Wave-induced turbulence ; Vertical mixing ; Mixed layer; Ekman currents 


\section{Introduction: direct injection of TKE}

Surface waves are known to be responsible for enhanced turbulence in the upper ocean (e.g. Agrawal et al., 1992), with strong expected effects on near-surface currents, temperature and other tracers (e.g. Mellor and Blumberg, 2004, Rascle and Ardhuin, 2009 and Takaya et al., 2011). This is important for many different applications, ranging from ocean remote-sensing to surface drift predictions and modelling of air-sea interactions for climate studies (e.g. Alford, 2003).

Recent progress in wave modelling now allows a realistic estimation of the full wave spectrum from the wind field alone. Such models include the dominant waves, that have been well modelled for some time (e.g. Janssen, 2008 and Rascle et al., 2008), but also the shorter waves (with frequencies up to $0.4 \mathrm{~Hz}$ at least) that significantly contribute to the wave-induced (Stokes) drift and to air-sea fluxes of momentum and energy (Ardhuin et al., 2009 and Ardhuin et al., 2010). Because such models are constrained to reproduce both dominant and short waves in a wide variety of conditions, the fluxes of energy in and out of the wave field that are provided by such models also have a fair chance of being realistic.

Following those improvements of wave modelling and aiming to improve upper-ocean understanding and modelling, the next issue is how to describe the wave-induced mixing in the upper-ocean and which properties of the wave field should be used.

This wave-induced turbulence and mixing on the upper ocean is believed to occur via three mechanisms: the breaking of waves (e.g. Agrawal et al., 1992 and Terray et al., 1996), the creation of Langmuir circulations by interaction with the currents (e.g. Craik and Leibovich, 1976 and Harcourt and D'Asaro, 2008), and perhaps a direct creation of turbulence by the orbital motion of non-breaking waves (e.g. Babanin and Haus, 2009 and Dai et al., 2010), although contrary evidence exists (Beyá, 2010). This paper will focus on the first mechanism, the mixing induced by wave-breaking, which is most likely the dominant source of turbulence in the upper few meters of the ocean. For the sake of simplicity, we will further refer to it as "the" wave-induced mixing.

The classical description for this wave-induced mixing 
comes with the model of Craig and Banner (1994), in which that mixing is taken into account by adding a flux of Turbulent Kinetic Energy (TKE) at the ocean surface, coming from the wave energy dissipation. In the near-surface region, the TKE diffuses downward and dissipates. Comparisons of this model with observed dissipation rates lead Craig and Banner (1994) and following authors (Terray et al., 1996, 2000; Soloviev and Lukas, 2003; Gemmrich and Farmer, 2004) to prescribe large near-surface diffusion (through values of the roughness length of the order of the significant wave height $H_{s}$ ); otherwise the TKE does not penetrate deep enough. This description of waveinduced mixing has been widely used for diverse applications, ranging from mixed layer depth analysis (e.g. Noh, 1996; Mellor and Blumberg, 2004) to surface currents (Stacey, 1999; Rascle and Ardhuin, 2009) and turbulence (Noh et al., 2004). However the exact link between this surface diffusion and the sea state parameters (such as the significant wind-sea wave height) is still not precisely known and remains the major uncertainty of this description.

Contrary to the model of Craig and Banner (1994), the model of Kudryavtsev et al. (2008) follows the idea of direct depth injection of turbulence by breaking waves (Kitaigorodskii, 1984; Terray et al., 1996; Donelan, 1998; Sullivan et al., 2004). The near-surface TKE balance is then between this injection and the dissipation, with the diffusion only playing a minor role. Very small near-surface diffusion is then possible in the model of Kudryavtsev et al. (2008). Physically, it means that the molecular viscous sublayer is disrupted by passing breakers but recovers afterwards. Kudryavtsev et al. (2008) argued that this description agrees with observations of TKE dissipation while it also agrees with "cold skin" and viscous sublayer observations at the surface. By getting rid of the large and poorly constrained roughness length at the surface of the Craig and Banner's model, their model proposes a description of the nearsurface turbulence based only on the size and spectral distribution of breaking waves.

A similar approach of direct depth injection of wave energy has been undertaken in the more sophisticated models of Sullivan et al. (2004) and Sullivan et al. (2007). Those models, instead of using simplified TKE equations, aim to resolve most breaking events and their effects on the mean and turbulent properties of the flow. They use Direct Numerical Simulations (DNS) or Large Eddy Simulations (LES) and resolve the depth injection of momentum by a distribution of breaking waves. As in the model of Kudryavtsev et al. (2008), the near-surface turbulence only depends on the size and spectral distribution of breaking waves.

Even though the two aforementioned models follow the same idea on a direct injection mechanism, they show large differences. We review here those differences and compare to available observations. On the one hand the model of Kudryavtsev et al. (2008) injects TKE reasonably well compared to TKE dissipation observations, but the depth reached by each breaking wave as well as the spectral distribution of breaking waves are unrealistic, especially for developed seas. On the other hand the model of Sullivan et al. (2007) uses more realistic breakers, but their spectral distribution is displaced towards small scales, and as a result, the TKE injection is far too shallow compared to the TKE dissipation observations, especially for developed seas.

The paper is organized as follows: the injection of TKE is described in section 2, and the corresponding TKE dissipation rates are shown in section 3. A discussion of the discrepancies and uncertainties follows in section 4 .

\section{Parameterizations of TKE injection}

We adopt here a turbulence closure following the widely used model of Mellor and Yamada (1982), level 2.5. The equations for the TKE evolution (Craig and Banner, 1994; Kudryavtsev et al., 2008, eq. 3.7) may be written as

$$
\begin{aligned}
\frac{\partial b}{\partial t}= & \underbrace{\frac{\partial}{\partial z}\left(l q \frac{S_{q}}{S_{m}} \frac{\partial b}{\partial z}\right)}_{a}+\underbrace{l q\left(\frac{\partial \mathbf{u}}{\partial z}\right)^{2}}_{b} \\
& -\underbrace{\tau^{\mathbf{w}} \frac{\partial \mathbf{u}}{\partial z}}_{c}-\underbrace{\frac{q^{3}}{l}}_{d}+\underbrace{P^{w b}(z)}_{e},
\end{aligned}
$$

where $\mathrm{q}$ is the turbulent velocity scale, $b=(\mathrm{q} / \mathrm{Sm})^{2} / 2$ is the TKE, $\mathbf{u}$ is the mean velocity, $\mathrm{z}$ is the upward vertical coordinate, 1 is the mixing length, $l q S_{q} / S_{m}$ is the eddy diffusivity where $S_{m}$ and $S_{q}$ are model constants for which the conventional values are 0.39 and 0.2 (Mellor and Yamada, 1982).

Contrary to the model of Craig and Banner (1994) where the TKE input is at the surface, we add here the term $P^{w b}(z)$, the volume injection of TKE by wave-breaking. $\tau^{\mathbf{w}}$ is the vertical momentum flux supported by the wave motion, which is included here for completeness but is unimportant because it is associated with a small TKE production.

As discussed by Kudryavtsev et al. (2008), the TKE balance in the near-surface layer is primarily between dissipation (d) and the wave TKE injection (e), with a small role of diffusion (a) (fig. 1). The TKE production by the work of the turbulent momentum flux on the current (b) and by the work of the wave momentum flux on the current (c) is smaller.

Fig. 1

The TKE injection is distributed along phase speed and depth as

$$
P^{w b}(z)=\int_{c} f_{c}(z) D_{E}(c) d c,
$$

where $D_{E}(c)$ is the energy lost by breaking waves with phase speed between $c$ to $c+d c$, and where $f_{c}(z)$ is a normalized function of depth, which represents the injection by each individual breaking wave.

The profile of TKE injection depends on the choice of parameterizations of $f_{c}(z)$ and of $D_{E}(c)$.

\subsection{The vertical profile of monochromatic breakers}

Each monochromatic breaker is supposed to inject TKE to a depth proportional to its wavelength. Kudryavtsev et al. (2008) used a rectangular function for $f_{c}(z)$,

$$
f_{c}^{\text {rect }}(z)= \begin{cases}k & \text { for }-1 / k<z<0, \\ 0 & \text { below, }\end{cases}
$$




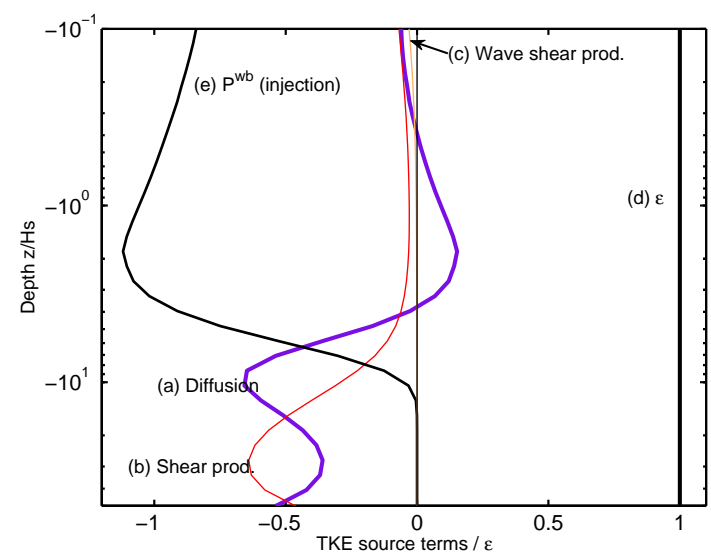

Figure 1: Balance of the TKE terms in a typical model calculation. Each term is normalized by the dissipation $\epsilon$. The model of Craig and Banner (1994) is used with a roughness length of $0.008 \mathrm{~m}$ as in Kudryavtsev et al. (2008). The wind is set to $10 \mathrm{~m} \mathrm{~s}^{-1}$ and the waves are fully developed. The TKE injection uses the wind growth $\beta_{P}$ and the vertical profile is $f_{c}^{\text {rect }}(z)$ (see further). Note that diffusion is generally small except immediately below the injection layer.

where $k=g / c^{2}$ is the wavenumber. This corresponds to a linear decrease of the TKE flux from its surface value to zero at a depth of $-1 / k$.

On the contrary, Sullivan et al. (2004) and Sullivan et al. (2007) proposed an analytical breaker momentum impulse based on observed properties of breaking waves in laboratory measurements (Melville et al., 2002). This analytical breaker integrates in time and horizontal dimensions to (see also Rascle et al., 2006)

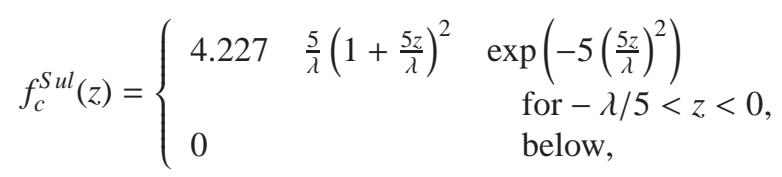

where $\lambda=2 \pi / k$ is the wavelength.

This injection profile (4) is much shallower than the rectangular profile (3) of Kudryavtsev et al. (2008) (fig. 2). For instance, $80 \%$ of the energy is released above a depth of the order of $k z \sim 0.35$, i.e. $z / \lambda \sim 0.055$. This profile is in agreement with the recent laboratory measurements of Drazen and Melville (2009, their fig. 5 right), where most of the energy is released at $0<z / \lambda<0.075$ within 3 wave periods after the breaking event, and only a few orders of magnitude less energy reaches depths $0.075<z / \lambda<0.15$ after 12 wave periods.

Fig. 2

\subsection{The spectral distribution of breakers}

\subsubsection{In the model of Kudryavtsev et al. (2008)}

The spectral distribution of dissipation is usually chosen as equal to the spectral distribution of the wind input,

$$
D_{E}(c)=\beta \omega E(c),
$$

where $\beta$ is the wind wave growth rate, $\omega$ is the radian frequency, and $E(c)$ is the energy spectrum.

Kudryavtsev et al. (2008) chose for the energy spectrum a simple saturation spectrum with a high-speed cut-off at the

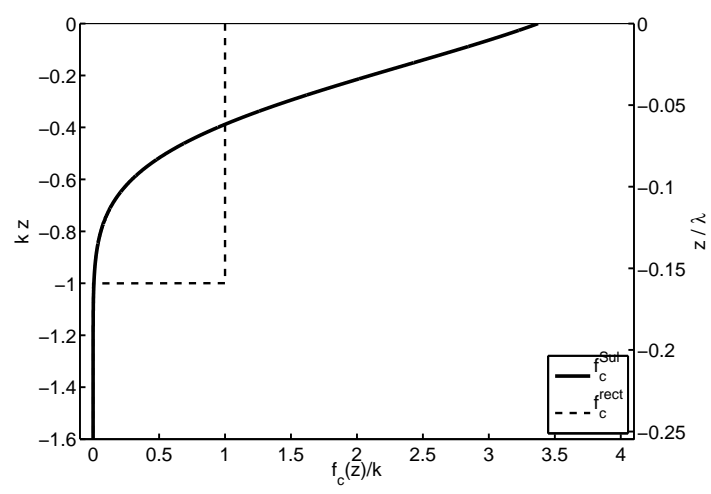

Figure 2: Vertical profile of monochromatic breakers, comparison of the injection profile (4) of Sullivan et al. (2004) and of the rectangular function (3) of Kudryavtsev et al. (2008).

spectral peak $c_{p}$. With equivalent results ${ }^{1}$, we use in this paper the complete spectral shape of Kudryavtsev et al. (1999) in order to avoid this sharp cut-off at $c_{p}$.

Kudryavtsev et al. (2008) applied to this energy spectrum the growth rate of Plant (1982), which may be written as

$$
\beta_{P}=c_{\beta}\left(\frac{u_{*}}{c}\right)^{2}
$$

where $u_{*}$ is the air-side friction velocity and $c_{\beta}$ is a numerical constant.

Fig. 3

The figure 3a shows the energy (dotted line) and energy input (dashed line) spectra as function of the normalized phase speed $c / c_{p}$, for a 10 -meter wind speed $U_{10}$ of $10 \mathrm{~m} \mathrm{~s}^{-1}$. The growth rate (6) predicts that the wind input is positive for waves approaching the peak phase speed. This feature is valid for young wave ages, but as waves get more developed, large waves approach the speed of the wind $\left(c / U_{10} \simeq 1\right)$ and therefore do not experience any work from the wind. To take this into account, we need to apply the growth rate of Makin and Kudryavtsev (1999), which, neglecting the sheltering effect of Hara and Belcher (2002), may be written as

$$
\left\{\begin{array}{l}
\beta_{M K}=c_{\beta}\left(\frac{u_{*}}{c}\right)^{2}\left(1-1.3\left(\frac{c}{U_{10}}\right)^{5}\right), \\
\beta_{M K} \geq 0
\end{array}\right.
$$

This predicts that the wind input drops to zero for waves approaching the speed of the wind $\left(c / U_{10} \simeq 0.95\right)$ contrary to the growth rate (6). For developed sea states (see fig. 3a color solid lines), the growth rate (7) induces a shift of the breaking waves towards smaller waves.

\footnotetext{
${ }^{1}$ We also performed all the following calculations with a saturated cut-off spectra as in Kudryavtsev et al. (2008) and found only small differences. The reason is that this saturated cut-off spectra has no energy at frequencies lower than the spectral peak but compensates with higher energy at frequencies immediately higher the peak. Consequently, for the sake of simplicity, we will only discuss here the results with the complete spectrum of Kudryavtsev et al. (1999).
} 


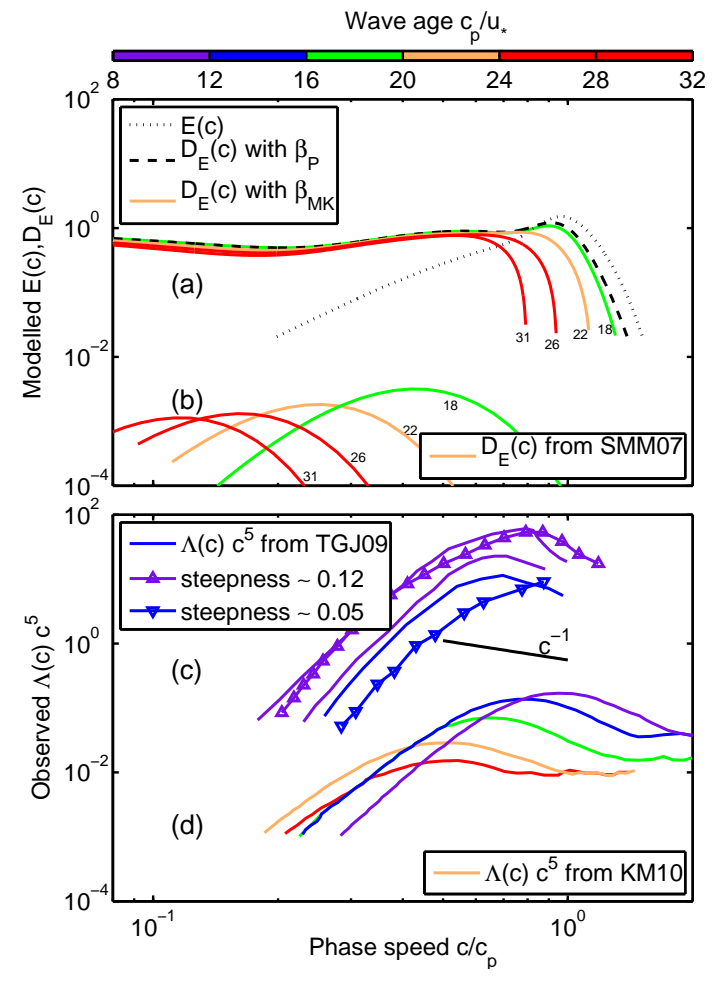

Figure 3: Spectral distribution of energy dissipation $D_{E}(c) \mathrm{dc}$ in the phase speed space. Note the use of $\log$ scales, that the phase speed $c$ is normalised by the peak phase speed $c_{p}$ on the x-axis and that each curve is arbitrarily shifted in the y direction, i.e. only the relative distribution along phase speed is represented here. From top to bottom: (a) Energy spectrum $E(c)$ (black dotted line) and energy input spectra $D_{E}(c)$ calculated with the distinct growth rates (6) of Plant (1982) (black dashed line) and (7) of Makin and Kudryavtsev (1999) (color solid lines). (b) Energy dissipation $D_{E}(c)$ (9) inferred from the statistical analysis of Sullivan et al. (2007), denoted as SMM07. In (a,b) the wind is set to $10 \mathrm{~m} \mathrm{~s}^{-1}$ and the wave age $c_{p} / u_{*}$ varies in the range $18-31$. Black color indicates no wave age variation. (c,d) Fifth moment of breaking distribution $c^{5} \Lambda(c)$ observed by (c) Thomson et al. (2009, their fig. 3b) and by (d) Kleiss and Melville (2010, their fig. 4c). In (c) the wave age is in the range $8<c_{p} / u_{*}<15$ but further partition is only estimated since Thomson et al. (2009) organized their data according to wave steepness $a k_{p}$.

\subsubsection{In the model of Sullivan et al. (2007)}

Sullivan et al. (2007) did not use any consideration on the spectral distribution of energy or energy input. Instead, they distributed the breakers along wavenumbers according to statistics of wave breaking crest lengths per unit area (the function $\Lambda(c)$, see Phillips, 1985) observed by Melville and Matusov (2002). Namely, they supposed that the probability density $P(c) d c$ of the breaking events decreases exponentially with the phase speed $c$ of the breaker,

$$
P(c) \propto \exp \left(-b_{2} \frac{c}{u_{*}}\right),
$$

where $b_{2}$ is a numerical constant dependent on the wave age and determined by considerations of the total energy and momentum wave to ocean fluxes. As the energy released by their 3-dimensional breaker increases with $c^{8}$, the spectral distribution of dissipation associated with their breaking statistics is (Phillips, 1985, eq. 6.4)

$$
D_{E}(c) d c \propto P(c) c^{8} d c .
$$

As a result of this distribution, only small waves dissipate energy through breaking (fig. $3 \mathrm{~b}$ ), with the dominant phase speed $c_{E}$ around $0.1-0.4$ times the peak phase speed $c_{p}$ (see also Sullivan et al., 2007, their fig. 3). In fig. 3b we take a Philips parameter $r=0.2$ for the high frequency cut-off and values $b_{2}=$ $(2.196,1.86,1.46,1.06)$ for wave ages $c_{p} / u_{*}=(31,26,22,18)$ as in Sullivan et al. (2007).

\subsubsection{In the recent observations of breaking statistics}

The exponential decay (8) was inferred from the airborne observations of Melville and Matusov (2002). Additional observations have been made more recently by Gemmrich et al. (2008), Thomson et al. (2009) and Kleiss and Melville (2010). The latter two are reproduced in fig. $3 \mathrm{c}$ and fig. $3 \mathrm{~d}$, denoted as TGJ09 and KM10. A $c^{-1}$ slope would be expected in the equilibrium range (Phillips, 1985) but it is only approximately found for larger waves (Kleiss and Melville, 2010). A $c^{0}$ slope would be expected in the saturation range. As waves get more developed, the shift towards breaking waves smaller than the peak waves seems to be confirmed by the data of Kleiss and Melville (2010). That shift was absent from the analysis of Kudryavtsev et al. (2008) but appears if we correct their growth rate with $\beta_{M K}$ (see fig. 3a). That shift is present in the model of Sullivan et al. (2007) but the whole dissipation occurs at much smaller scales (see fig. 3b).

\section{TKE dissipation rates}

The TKE injection is calculated using (2) and different vertical profiles $f_{c}(z)$ of individual breakers, different spectral distributions $D_{E}(k)$ of wave dissipation and different wave ages. The results are then compared to observations of TKE dissipation ${ }^{2}$.

\subsection{In the observations}

Measurements of TKE by Terray et al. (1996), Drennan et al. (1996), Anis and Moum (1995), Soloviev and Lukas (2003) and Gemmrich and Farmer (2004) are shown in fig. 4, along with wave age information when available. The first three sets of data are consistent and show no wave age trend. Data collected during TOGA-CORE by Soloviev and Lukas (2003) show shallower TKE, and data collected by Gemmrich and Farmer (2004) shows a more enhanced dissipation close to the surface.

\section{Fig. 4}

\subsection{In the model of Kudryavtsev et al. (2008)}

Fig. 5a shows the profiles of TKE dissipation obtained with the joint use of the rectangular function $f_{c}^{\text {rect }}(z)$ and of the growth rate $\beta_{P}$, as made by Kudryavtsev et al. (2008) . The TKE is injected quite deeply (black line) and compares well with observations (thick dashed line).

\footnotetext{
${ }^{2}$ Note that we did not suppose an equilibrium between injection and dissipation of TKE but that we computed the additional diffusion of turbulence according to the model of Craig and Banner (1994) with a roughness length of $0.008 \mathrm{~m}$ as in Kudryavtsev et al. (2008). That diffusion is generally small except immediately below the injection layer (see fig. 1).
} 


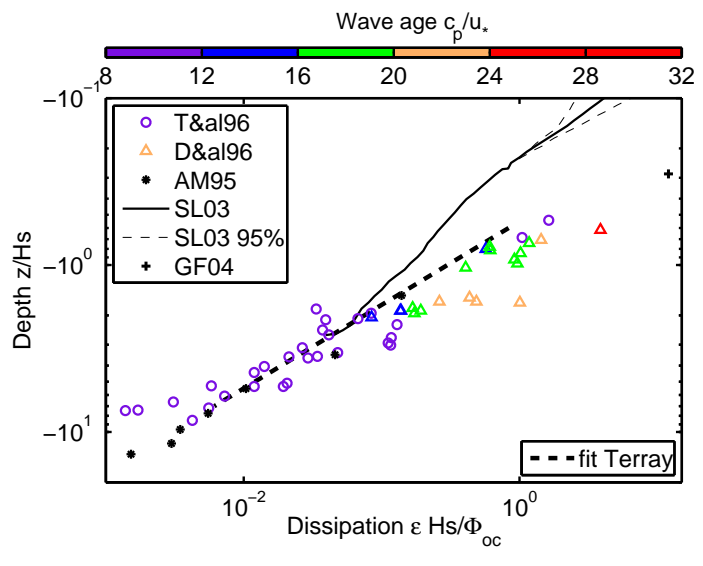

Figure 4: Observed TKE dissipation rates normalized by its integral over depth $\Phi_{o c}$ and $H_{s}$, as function of depth normalized by $H_{s}$. Data shown are taken from Terray et al. (1996, 2000), Drennan et al. (1996), Anis and Moum (1995), Soloviev and Lukas (2003) (with the 95\% confidence interval shown in thin dashed lines) and Gemmrich and Farmer (2004, their deployment II). The thick dashed line corresponds to the $z^{-2}$ fit of Terray et al. (1996). Colors indicate the wave age, with black color for no wave age information.

When the breaker injection profile $f_{c}^{S u l}(z)$ is used instead of the rectangular profile (3), the model dissipation distribution gets accordingly shallower (purple solid line). Furthermore, when the waves become developed, the growth rate $\beta_{M K}$ gradually departs from $\beta_{P}$ and shifts the breaking waves towards smaller scales, as shown in the previous section. The resulting TKE dissipation becomes accordingly shallower (red solid line), with dissipation rates 3 times less than the observations fit of Terray et al. (1996).

\section{Fig. 5}

\subsection{In the model of Sullivan et al. (2007)}

The figure $5 \mathrm{~b}$ shows the profiles of TKE dissipation obtained with the joint use of the profile $f_{c}^{S u l}(z)$ and with the statistical distribution $D_{E}(k)$ given by (9), as made by Sullivan et al. (2007). The TKE injection is very shallow (color solid line) and does not compare with observations (thick dashed line).

Indeed, with each monochromatic breaker injecting TKE to a depth $z \sim 1 / k$, depths of elevated dissipation rates scale with $c_{E}^{2} / g$ (see Sullivan et al., 2007, their fig. 11), whereas observations of Drennan et al. (1996) scale with $c_{p}^{2} / g$, which is 1 to 2 orders of magnitude larger. The injection of turbulence with the statistical distribution (9) is thus extremely shallow, around only $1 \mathrm{~m}$ for a wind speed of $15 \mathrm{~m} \mathrm{~s}^{-1}$ (see also Sullivan et al., 2007, their fig. 4). When the waves are developed, we find that the agreement is recovered with observed TKE dissipation if each monochromatic breaker injects to a depth of $10 / k$ (fig. $5 \mathrm{~b}$, thin dashed red curve), which is unrealistically more than 10 times deeper than the injection (4) inferred from laboratory measurements.

Although the spectral distribution of breakers chosen by Sullivan et al. (2007) is physically sound, with a shift of the breaking waves towards higher frequency as the waves develop, it leads to a poor agreement with observed TKE dissipation rates. This results both from the shallow injection depth of individ-

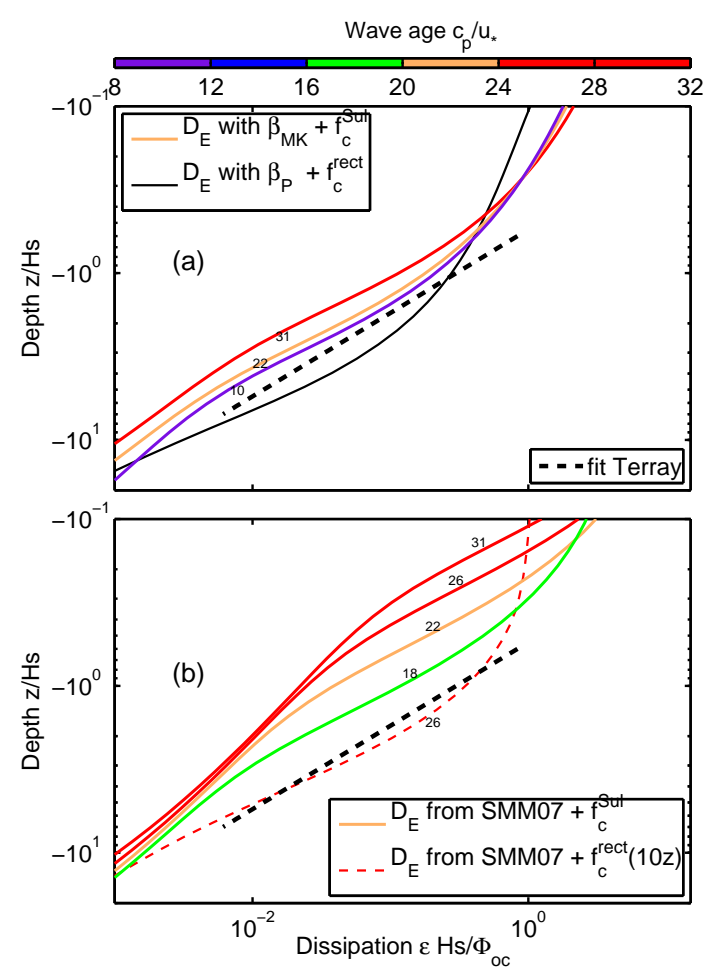

Figure 5: Modelled TKE dissipation rates normalized by its integral over depth $\Phi_{o c}$ and $H_{s}$, as function of depth normalized by $H_{s}$. The wind is set to $10 \mathrm{~m} \mathrm{~s}^{-1}$ and the wave age varies in the range $10<c_{p} / u_{*}<31$. The thick dashed line corresponds to the $z^{-2}$ fit of Terray et al. (1996). (a) According to the analysis of Kudryavtsev et al. (2008), the solid black line uses the rectangular profile $f_{c}^{r e c t}(z)$ and the growth rate $\beta_{P}$. The black is used because there is no variation with the wave age. Color solid lines use the injection profile $f_{c}^{S u l}(z)$ and the growth rate $\beta_{M K}$ (eq. 7). (b) According to the analysis of Sullivan et al. (2007), denoted as SMM07, color solid lines use injection profile $f_{c}^{S u l}(z)$ and the statistical energy dissipation (9). Red dashed line shows the dissipation for developed waves $\left(c_{p} / u_{*}=26\right)$ but with an (unrealistic) breaker injection profile 10 times deeper.

ual breakers and from their spectral distribution, which imposes that only the very short waves contribute to the dissipation. As a striking illustration, the analysis of Sullivan et al. (2007) predicts that turbulence reaches greater depth for young sea states than for mature ones. This is not supported by the scaling of depth by $H_{s}$ in the dissipation measurements (e.g. Terray et al., 1996), which means that in the observations, mature seas inject turbulence deeper than young seas.

\section{Discussion}

The previous section highlights the uncertainties that remain to physically describe the turbulence close to the surface, according to the different observations of TKE dissipation, breaking waves and momentum injection. Even in two of the most recent models of near-surface dynamics, the impact of wavebreaking over depth is not known to the zeroth order and no consensus has been reached yet.

Can we identify the source of discrepancy? Are the observations of TKE dissipation reliable, even for developed seas? What is the likely spectral distribution of breakers at the ocean 
surface? Can the observations of individual breaker dissipation be extended to a spectral distribution of breakers using a self similarity hypothesis?

\subsection{Measurements of TKE dissipation}

The scatter remains large between the different measurements, and many reasons can be invoked.

First, the measurements by Terray et al. (1996) were obtained from a fixed tower, whereas measurements by Drennan et al. (1996) and Soloviev and Lukas (2003) were obtained from ship-mounted devices. For measurements at fixed location, the essential Taylor's frozen turbulence hypothesis to calculate the turbulence wavenumber spectrum from the frequency spectrum is not satisfied, and the extension to unsteady advection (Lumley and Terray, 1983) brings additional uncertainties compared to ship-mounted measurements. On the other hand, Gemmrich and Farmer (2004) used direct spatial measurements with an acoustic Doppler profiler mounted on a surface-following float, and no transformation of a frequency spectrum into a wavenumber spectrum was required.

Second, the dissipation profile is different whether the timeaveraging is made at fixed location or with a surface-following reference. We can make a comparison with the case of the velocity. In a fixed reference frame, the wave orbital velocities average to zero everywhere below the wave through, and the Stokes drift awkwardly appears between crest and trough. A natural description of the Stokes drift involves, before time averaging the orbital velocities, a coordinate change in the vertical direction (Mellor, 2003) or in vertical-horizontal dimensions (Andrews and McIntyre, 1978; Ardhuin et al., 2008). If, on the contrary, the correlations between surface elevation and orbital velocities are disregarded, the mean velocities obtained are unrelated to the true Lagrangian Stokes drift. It should be emphasized here that a similar description with coordinate change would be natural as well for the near-surface turbulence, as it appears also strongly correlated to the wave phase (Veron et al., 2009; Gemmrich, 2010). Description in coordinates stretched by the waves might possibly see a distortion of turbulence by the waves (Teixeira and Belcher, 2002; Ardhuin and Jenkins, 2006).

Third, the profiles of TKE dissipation are scaled with the overall energy flux $\Phi_{o c}$ from waves to the ocean. That energy flux is usually obtained by estimating the wind input with an empirical formulation like (7) (Terray et al., 1996; Drennan et al., 1996; Soloviev and Lukas, 2003; Gemmrich and Farmer, 2004). The consistency of that estimation with the integral over depth of extrapolated observed dissipation profiles is essential. Gemmrich (2010) recently noted that such extrapolation might be erroneous in fixed frame measurements limited below the trough level, since high turbulence levels were found highly intermittent and localized essentially above the trough level.

Fourth, a significant fraction of the near-surface dissipation takes place in the breaking surface waves saturated by airbubbles. Soloviev and Lukas (2003) found up to $80 \%$ of turbulence dissipation missing, possibly due to removal of bubble distorted measurements. Unfortunately, none of the existing sensor technologies have been able to provide turbulence measurements within an active breaking wave due to high concentration of air-bubbles. Soloviev et al. (2012) have recently suggested using high-resolution 3D sonar technology for estimating turbulence characteristics of breaking waves by measuring the geometrical properties of bubble clouds in the edge detection mode. This technology, however, has only been tested in turbulent bubble-cloud wakes produced by surface ships.

\subsection{Spectral distribution of breakers}

As discussed in section 2.2, there is a large uncertainty on the main scale supporting energy dissipation. Melville and Matusov (2002) observed the rapid decrease (8) of the breakers probability when their size increases. Those observations led Sullivan et al. (2007) to propose a spectral distribution of breakers where the energy dissipation is supported mainly by the small waves $\left(c / c_{p} \sim 0.1-0.4\right)$. More recent observations by Gemmrich et al. (2008), Thomson et al. (2009) and Kleiss and Melville (2010) seem to show that the decrease (8) only occurs at scales around the spectral peak. As a result, even if most of the breakers occur at small scales $\left(c / c_{p} \sim 0.2-0.5\right)$, the actual energy transfer rather occurs at larger scales $\left(c / c_{p} \sim 0.5-1\right)$, of the order of the dominant waves (see Thomson et al., 2009, their fig. 3).

Kleiss and Melville (2011), using two different image processing methods, were able to qualitatively reproduce the results of Melville and Matusov (2002) and of Gemmrich et al. (2008), and argue in favor of the latter. Consistently, this study highlights that the turbulence induced by the distributions of Melville and Matusov (2002) and Sullivan et al. (2007) contradicts observations of TKE dissipation profiles, and furnishes indirect evidence that such distributions of wave breaking might be unrealistic.

\subsection{Breaker injection profiles and self similarity hypothesis}

Unless new dissipation measurements are made in developed seas and reveal shallower effects of wave breaking, we are led to question the fundamental assumption of breaker self similarity. The laboratory measurements of Melville et al. (2002) and Drazen and Melville (2009) should not be valid for wave breaking at any scale. In particular, a wave of wavenumber $k$ should not break in the same manner when it is the dominant wave as when it breaks around the crest of a longer wave. Additional parameters including the wave slope (Melville and Rapp, 1985; Banner and Peirson, 2007; Drazen et al., 2008) are necessary to determine the onset of wave breaking, and are likely to have an impact on the TKE injection profile of the breakers.

\section{Concluding remarks}

The recent models of Sullivan et al. (2007) and Kudryavtsev et al. (2008) represent the wave-induced near-surface turbulence by an equilibrium between dissipation and direct volume injection of TKE by wave breaking. Diffusion of TKE only plays a minor role in the near-surface TKE budget. 
In this study, we review those models in terms of available measurements of turbulence induced by single breakers in the laboratory, in terms of the observed spectral distribution of breakers in the ocean and in terms of the observed profiles of TKE dissipation in the ocean.

Good agreement was obtained by Kudryavtsev et al. (2008) with observations of TKE dissipation. We show here that this agreement is weakened with regards to two properties: First, their monochromatic breaker injects TKE two times deeper than the laboratory measurements of Melville et al. (2002) and Drazen and Melville (2009). Second, when wind-waves get developed, observations (e.g. Banner et al., 2000; Gemmrich et al., 2008) show that large waves at the spectral peak stop breaking, inducing a shift of the dissipation towards intermediate shorter waves.

On the other hand, the model of Sullivan et al. (2007) uses a realistic monochromatic breaker, and uses a spectral distribution in which only very small waves break. As a consequence, their TKE injection is far too shallow compared to observed dissipation profiles.

A complete and coherent description of the near-surface turbulence is thus still missing. This study might however help to foresee what the general picture will be in the near future. For young sea states, energy dissipation is likely carried by the dominant waves, leading to approximate agreement with TKE dissipation profiles previously observed. For developed waves, energy dissipation likely shifts towards intermediate shorter waves, but evidence of a shift of TKE dissipation towards shallower depth is still missing.

Should that shift in dissipation fail to be confirmed in future measurements, then it should be questioned whether another physical mechanism is missing to inject TKE much deeper for developed seas. Such mechanism could involve a revision of the breakers self-similarity hypothesis or a coupling between long unbreaking waves and short breaking waves.

Acknowledgments. Critical comments from V. Kudryavtsev were useful throughout the writing of this paper. Comments from the two anonymous reviewers were also useful to improve the presentation. N.R. acknowlegdes the financial support of CNRS/INSU and the help from M. Rouault at the University of Cape Town. This research would not have been finalized without the support of CNES and of the ANR through the REDHOTS project.

\section{References}

Agrawal, Y. C., Terray, E. A., Donelan, M. A., Hwang, P. A., Williams, A. J., Drennan, W., Kahma, K., Kitaigorodskii, S., 1992. Enhanced dissipation of kinetic energy beneath breaking waves. Nature 359, 219-220.

Alford, M., 2003. Improved global maps and 54-years old history of wind-work on ocean inertial motions. Geophys. Res. Lett. 30 (8).

Andrews, D. G., McIntyre, M. E., 1978. An exact theory of nonlinear waves on a Lagrangian-mean flow. J. Fluid Mech. 89, 609-646.

Anis, A., Moum, J. N., 1995. Surface wave-turbulence interactions: Scaling $\varepsilon(z)$ near the sea surface. J. Phys. Oceanogr. 25, 2025-2045.

Ardhuin, F., Jenkins, A. D., 2006. On the interaction of surface waves and upper ocean turbulence. J. Phys. Oceanogr. 36 (3), 551-557.

Ardhuin, F., Marié, L., Rascle, N., Forget, P., Roland, A., 2009. Observation and estimation of Lagrangian, Stokes and Eulerian currents induced by wind and waves at the sea surface. J. Phys. Oceanogr 39, 2820-2838.
Ardhuin, F., Rascle, N., Belibassakis, K., 2008. Explicit wave-averaged primitive equations using a generalized lagrangian mean. Ocean Modelling 20 (1), 35-60.

Ardhuin, F., Rogers, E., Babanin, A., Filipot, J., Magne, R., Roland, A., van der Westhuysen, A., Queffeulou, P., Lefevre, J., Aouf, L., et al., 2010. Semiempirical dissipation source functions for ocean waves. part i: Definition, calibration, and validation. Journal of Physical Oceanography 40 (9), 1917 1941.

Babanin, A., Haus, B., 2009. On the existence of water turbulence induced by nonbreaking surface waves. Journal of Physical Oceanography 39 (10), 2675-2679.

Banner, M. L., Babanin, A. V., Young, I. R., 2000. Breaking probability for dominant waves on the sea surface. J. Phys. Oceanogr. 30, 3145-3160. URL http://ams . allenpress . com/archive/1520-0485/30/12/pdf/i1520-0

Banner, M. L., Peirson, W. L., 2007. Wave breaking onset and strength for two-dimensional deep-water wave groups. J. Fluid Mech. 585, 93-115.

Beyá, J., 2010. On the interaction of ocean waves and turbulence. Master's thesis, School of Civil and Environmental Engineering, University of New South Wales.

URL http: //unsworks . unsw . edu . au/fapi/datastream/unsworks : 9758/SOU

Craig, P. D., Banner, M. L., 1994. Modeling wave-enhanced turbulence in the ocean surface layer. J. Phys. Oceanogr. 24, 2546-2559.

URL http: //ams . allenpress . com/archive/1520-0485/24/12/pdf/i1520-0

Craik, A. D. D., Leibovich, S., 1976. A rational model for Langmuir circulations. J. Fluid Mech. 73, 401-426.

Dai, D., Qiao, F., Sulisz, W., Han, L., Babanin, A., 2010. An experiment on the nonbreaking surface-wave-induced vertical mixing. Journal of Physical Oceanography 40 (9), 2180-2188.

Donelan, M. A., 1998. Air-water exchange processes. In: Imberger, J. (Ed.), Physical Processes in Lakes and Oceans. American Geophysical Union, Washington, D.C., pages 18-36, iSBN 0-87590-268-5.

Drazen, D., Melville, W., 2009. Turbulence and mixing in unsteady breaking surface waves. Journal of Fluid Mechanics 628, 85-119.

Drazen, D. A., Melville, W. K., Lenain, L., 2008. Inertial scaling of dissipation in unsteady breaking waves. J. Fluid Mech. 611, 307-332.

Drennan, W. M., Donelan, M. A., Terray, E. A., Katsaros, K. B., 1996. Oceanic turbulence dissipation measurements in SWADE. J. Phys. Oceanogr. 26, 808-815.

Gemmrich, J., 2010. Strong Turbulence in the Wave Crest Region. Journal of Physical Oceanography 40 (3), 583-595.

Gemmrich, J., Banner, M., Garrett, C., 2008. Spectrally resolved energy dissipation rate and momentum flux of breaking waves. Journal of Physical Oceanography 38 (6), 1296-1312.

Gemmrich, J. R., Farmer, D. M., 2004. Near-surface turbulence in the presence of breaking waves. J. Phys. Oceanogr. 34, 1067-1086.

Hara, T., Belcher, S. E., 2002. Wind forcing in the equilibrium range of windwave spectra. J. Fluid Mech. 470, 223-245.

Harcourt, R., D'Asaro, E., 2008. Large-eddy simulation of langmuir turbulence in pure wind seas. Journal of Physical Oceanography 38 (7), 1542-1562.

Janssen, P. A. E. M., 2008. Progress in ocean wave forecasting. J. Comp. Phys. 227, 3572-3594.

Kitaigorodskii, S. I., 1984. On the fluid dynamical theory of turbulent gas transfer across an air-sea interface in the presence of breaking wind waves. J. Phys. Oceanogr. 14, 960-972.

Kleiss, J., Melville, W., 2010. Observations of wave breaking kinematics in fetch-limited seas. Journal of Physical Oceanography 40 (12), 2575-2604.

Kleiss, J., Melville, W., 2011. The analysis of sea surface imagery for whitecap kinematics. Journal of Atmospheric and Oceanic Technology 28 (2), 219243.

Kudryavtsev, V., Shrira, V., Dulov, V., Malinovsky, V., 2008. On the vertical structure of wind-driven sea currents. Journal of Physical Oceanography 38 (10), 2121-2144.

Kudryavtsev, V. N., Makin, V. K., Chapron, B., 1999. Coupled sea surfaceatmosphere model. 2. spectrum of short wind waves. J. Geophys. Res. 104, 7625-7639.

Lumley, J. L., Terray, E. A., 1983. Kinematics of turbulence convected by a random wave field. J. Phys. Oceanogr. 13, 2000-2007.

Makin, V. K., Kudryavtsev, V. N., 1999. Coupled sea surface-atmosphere model. 1. wind over wave coupling. J. Geophys. Res. 104 (C4), 7613-7623.

Mellor, G., 2003. The three-dimensional current and surface wave equations. J. Phys. Oceanogr. 33, 1978-1989, corrigendum, vol. 35, p. 2304, 2005, see 
also Ardhuin et al., vol. 38, 2008.

Mellor, G., Blumberg, A., 2004. Wave breaking and ocean surface layer thermal response. J. Phys. Oceanogr. 34, 693-698.

Mellor, G. L., Yamada, T., 1982. Development of a turbulence closure model for geophysical fluid problems. Rev. Geophys. Space Phys. 20 (C2), 851875.

Melville, W., Rapp, R., 1985. Momentum flux in breaking waves. Nature 317 (6037), 514-516.

Melville, W. K., Matusov, P., 2002. Distribution of breaking waves at the ocean surface. Nature 417, 58-63.

Melville, W. K., Verron, F., White, C. J., 2002. The velocity field under breaking waves: coherent structures and turbulence. J. Fluid Mech. 454, 203-233.

Noh, Y., 1996. Dynamics of diurnal thermocline formation in the oceanic mixed layer. J. Geophys. Res. 26, 2189-2195.

Noh, Y., Min, H. S., Raasch, S., 2004. Large eddy simulation of the ocean mixed layer: the effects of wave breaking and Langmuir circulation. J. Phys. Oceanogr. 34, 720-733.

Phillips, O. M., 1985. Spectral and statistical properties of the equilibrium range in wind-generated gravity waves. J. Fluid Mech. 156, 505-531.

Plant, W. J., 1982. A relationship between wind stress and wave slope. J. Geophys. Res. 87, 1961-1967.

Rascle, N., Ardhuin, F., 2009. Drift and mixing under the ocean surface revisited: Stratified conditions and model-data comparisons. J. Geophys. Res. 114, C02016, doi:10.1029/2007JC004466.

Rascle, N., Ardhuin, F., Queffeulou, P., Croizé-Fillon, D., 2008. A global wave parameter database for geophysical applications. part 1: wave-current-turbulence interaction parameters for the open ocean based on traditional parameterizations. Ocean Modelling 25, 154-171, doi:10.1016/j.ocemod.2008.07.006.

URL http: //hal . archives-ouvertes.fr/hal-00201380/

Rascle, N., Ardhuin, F., Terray, E. A., 2006. Drift and mixing under the ocean surface. a coherent one-dimensional description with application to unstratified conditions. J. Geophys. Res. 111, C03016, doi:10.1029/2005JC003004.

Soloviev, A., Lukas, R., 2003. Observation of wave-enhanced turbulence in the near-surface layer of the ocean during TOGA COARE. Deep Sea Res. I 50, 371-395.

Soloviev, A., Maingot, C., Agor, M., Nash, L., Dixon, K., 2012. 3d sonar measurements in wakes of ships of opportunity. Journal of Atmospheric and Oceanic TechnologyIn press.

Stacey, M. W., 1999. Simulation of the wind-forced near-surface circulation in Knight Inlet: A parameterization of the roughness length. J. Phys. Oceanogr. 29, 1363-1367.

Sullivan, P. P., McWilliams, J. C., Melville, W. K., 2004. The oceanic boundary layer driven by wave breaking with stochastic variability. part 1. direct numerical simulation. J. Fluid Mech. 507, 143-174.

Sullivan, P. P., McWilliams, J. C., Melville, W. K., 2007. Surface gravity wave effects in the oceanic boundary layer: large-eddy simulation with vortex force and stochastic breakers. J. Fluid Mech. 593, 405-452.

Takaya, Y., Bidlot, J., Beljaars, A. C. M., Janssen, P. A. E. M., 2011. Refinements to a prognostic scheme of skin sea surface temperature. J. Geophys. Res. 115, C06009.

Teixeira, M. A. C., Belcher, S. E., 2002. On the distortion of turbulence by a progressive surface wave. J. Fluid Mech. 458, 229-267.

Terray, E. A., Donelan, M. A., Agrawal, Y. C., Drennan, W. M., Kahma, K. K., Williams, A. J., Hwang, P. A., Kitaigorodskii, S. A., 1996. Estimates of kinetic energy dissipation under breaking waves. J. Phys. Oceanogr. 26, 792807.

Terray, E. A., Drennan, W. M., Donelan, M. A., 2000. The vertical structure of shear and dissipation in the ocean surface layer. In: Proc. Symp. on Air-Sea Interaction, Sydney. University of New South Wales, pp. 239-245.

Thomson, J., Gemmrich, J., Jessup, A., 2009. Energy dissipation and the spectral distribution of whitecaps. Geophys. Res. Lett 36, L11601.

Veron, F., Melville, W., Lenain, L., 2009. Measurements of ocean surface turbulence and wave-turbulence interactions. Journal of Physical Oceanography 39 (9), 2310-2323. 